\title{
Hepatocyte Injury Induced by Contrast-Enhanced Diagnostic Ultrasound
}

\author{
Douglas L. Miller, PhD (D), Xiaofang Lu, MD, Mario Fabiilli, PhD, Chunyan Dou, MD
}

Received August 10, 2018, from the Department of Radiology, University of Michigan Health System, Ann Arbor, Michigan, USA. Manuscript accepted for publication November 5, 2018.

This work was supported by Public Health Service grant HL110990, awarded by the US National Heart Lung and Blood Institute, Department of Health and Human Services. The information contained herein does not necessarily reflect the position or policy of the US government, and no official endorsement should be inferred.

Address correspondence to Douglas L. Miller, PhD, Department of Radiology, University of Michigan Health System, 3240A Medical Science Building I, 1301 Catherine St, Ann Arbor, MI 48109-5667 USA.

E-mail:douglm@umich.edu

Abbreviations

$A L T$, alanine aminotransferase; AST, aspartate aminotransferase; CEDUS, contrast-enhanced diagnostic ultrasound; MI, mechanical index; US, ultrasound

doi: $10.1002 /$ jum. 14883
Objectives-Contrast-enhanced diagnostic ultrasound (US) has a potential to induce localized biological effects. The potential for contrast-enhanced diagnostic US bioeffects in liver were researched, with guidance from a report by Yang et al (Ultrasonics 2012; 52:1065-1071).

Methods-Contact and standoff scanning was performed for 10 minutes with a diagnostic US phased array at $1.6 \mathrm{MHz}$ during bolus injection or infusion of a contrast agent at a high dose. The impact of the imaging on rat liver was investigated by measuring enzyme release, microvascular leakage, and staining of injured hepatocytes.

Results - The results showed liver enzyme release at 30 minutes, indicating liver injury, and elevated extraction of Evans blue dye, indicating microvascular leakage. In addition, Evans blue and trypan blue vital-staining methods revealed scattered stained cells within the US scan plane. For the Evans blue method, fluorescent cell counts in frozen sections were greatest for standoff exposure with contrast infusion. The count decreased strongly with depth for bolus injection, which was probably reflective of the high attenuation noted for this agent delivery method.

Conclusions - The results qualitatively confirmed the report by Yang et al and additionally showed hepatocyte vital staining. Research is needed to determine the threshold for the effects and the contrast agent dose response.

Key Words - contrast-enhanced diagnostic ultrasound; diagnostic ultrasound adverse effects; liver ultrasound imaging; ultrasonic cavitation biology

【 ltrasound (US) contrast agents are used to enhance images with poor vascular resolution or detail. ${ }^{1}$ Introduced in the 1990s, the agents have been approved for use in visualizing the left ventricle and aiding endocardial border delineation for patients with suboptimal echocardiograms. In addition, many other applications have been explored. Recently, contrast-enhanced diagnostic ultrasound (CEDUS) of the liver has been established as a useful method. ${ }^{2}$ The evaluation of incidental findings of focal liver lesions appears to be an especially valuable application. ${ }^{3-5}$

Ultrasound contrast agents are suspensions of stabilized microbubbles. For example, Definity (perflutren lipid microsphere injectable suspension; Lantheus Medical Imaging, Inc, North Billerica, MA) is a solution of lipids supplied in a vial with perflutren (perfluoropropane) gas, which is shaken (using a dedicated vial shaker) to produce a suspension of microbubbles with a lipid skin. Definity is no longer sold for research purposes, but a replacement 
contrast agent can be produced for research by using a formula mimicking the Definity formula, as described previously. ${ }^{6}$ The contrast agent microbubbles are coincidentally small enough to circulate with the blood and have sizes suitable for strong interaction with diagnostic US pulses. ${ }^{7}$ Diagnostic US pulses can activate the microbubbles sufficiently to nucleate US cavitation, a well-known mechanism for biological effects. The benchmark effect is injury to capillaries as the microbubbles pulsate violently. This leads to a progression of local damage with an increasing pulse pressure amplitude, including endothelial cell injury, capillary leakage, petechial hemorrhage, and injury to adjacent parenchymal cells. The resulting microlesion can then elicit fibrin formation and inflammatory responses. This potential for patient injury from CEDUS presents a safety issue, which must be addressed to ensure the performance of CEDUS without risk of cavitation-induced patient injury.

Microvascular injury and microlesions have been reported in several different tissues and organs, including the intestine, ${ }^{8}$ cremaster muscle, ${ }^{9}$ abdominal muscle, ${ }^{10}$ heart, ${ }^{11,12}$ kidney, ${ }^{13,14}$ and pancreas. ${ }^{15}$ The effects occur above specific pulse pressure amplitudes (ie, thresholds), and the threshold for petechial hemorrhage increases approximately in proportion to the US frequency in the kidney ${ }^{16}$ and heart, ${ }^{17}$ with minimal or no effects for CEDUS above about $5 \mathrm{MHz}$ within the limits of diagnostic US.

The liver has received relatively little research attention regarding the cavitation safety issue. Shigeta et $\mathrm{al}^{18,19}$ studied possible bioeffects in rat liver scanned with an Acuson Sequoia 512 diagnostic US machine (Siemens Medical Solutions, Mountain View, CA) with linear and curved arrays using a mechanical index (MI) value of 0.7 or 1.8 (an exposure index related to cavitation potential) with continuous scanning for 1 minute. A late-phase exposure (10 seconds) was also performed to scan microbubbles taken up by Kupffer cells 5 minutes after administration. The contrast agents Levovist (air filled with palmitic acid stabilization; Schering AG, Berlin, Germany) and DD-723 (later named Sonazoid, perfluorobutane with phosphatidyl serine stabilization; Daiichi Sankyo Co, Tokyo, Japan) were investigated using histology on light and electron microscopy. Endothelial injury and platelet aggregates were found in exposed rat livers. Vacuolization was seen in hepatocytes, and microbubbles were observed inside Kupffer cells, likely due to phagocytosis. In addition, increased platelet activation was observed for in vitro testing in US-plus-contrast agent groups. The exposures had two aspects, which would tend to minimize cavitational bioeffects: a relatively high US frequency (either 8 or $12 \mathrm{MHz}$ ) and continuous exposure (which can destroy microbubbles before they reach the focal region). The platelet activation seen in vitro, especially with Levovist, may not actually translate to the in vivo situation.

Yang et $\mathrm{al}^{20}$ used a Vivid 7 Dimension US machine (GE Vingmed Ultrasound, Horten, Norway) with an $\mathrm{S} 4$ transducer at $1.5 \mathrm{MHz}$ to evaluate the safety of US-targeted microbubble destruction (related to use of diagnostic US as a means for gene delivery to liver cells). The probe was handheld on the depilated abdomens of rats with a 3-cm image depth. Exposure to the liver was performed by alternating a low viewing MI of 0.08 and then switching to a "flash" mode at a high MI of 1.0, which gave alternating destruction and refill of microbubbles, and continued for 10 minutes. The contrast agent Zhifuxian (Xinqiao Hospital, Chongqing, China) was used as a $500-\mu \mathrm{L} / \mathrm{kg}$ bolus dose. This agent had lipid stabilization and perfluoropropane gas, which was prepared by shaking to yield 4 to $9\left(10^{9}\right) \mathrm{mL}^{-1}$ microbubbles of $2.1 \mu \mathrm{m}$ in mean diameter. ${ }^{21}$ Groups included control, agent only, US only, and US plus the agent with assessments at 0-, 0.5 -, 12-, and 24-hour time points. Evans blue dye was used as a tracer for vascular permeability in optical microscopy and tissue extraction, and lanthanum nitrate was used as a tracer for electron microscopy. In addition, the liver enzymes alanine aminotransferase (ALT) and aspartate aminotransferase (AST) were measured in serum samples as a measure of liver injury. The US-plus-microbubble groups had Evans blue extravasation into the parenchyma and lanthanum detectable in the cytoplasm. The AST and ALT levels were elevated at 0.5 hours but returned to normal at 24 hours. Overall, the results suggested that CEDUS increased capillary and cell membrane permeability with some hepatic toxicity.

The purpose of this study was to evaluate CEDUS-induced bioeffects in rat liver. The study by Yang et $\mathrm{al}^{20}$ was used as the initial model because it appears to be the only account of CEDUS-induced liver injury. Our GE Vivid 7 Dimension machine was used with our replacement contrast agent (similar to 
Definity). Results were generally supportive of the study by Yang et $\mathrm{al}^{20}$ and also indicated hepatocyte injury by Evans blue staining after application of intermittent exposure protocols similar to our previous heart and kidney research.

\section{Materials and Methods}

\section{Animal Preparation}

This in vivo research was conducted with the approval of the Institutional Animal Care and Use Committee at the University of Michigan. Male rats (Sprague Dawley; Charles River Laboratories, Wilmington, MA) with a mean weight of $317 \pm 36 \mathrm{~g}$ were used in this study, as detailed below. Six were lost from the study because of anesthetic death or technical problems. The rats were anesthetized via intraperitoneal injection of $40-\mathrm{mg} / \mathrm{kg}$ pentobarbital. The abdomen was shaved and depilated to maximize US transmission. A 24-gauge cannula was inserted into a tail vein for intravenous injections and infusion of the contrast agent. Rats were placed in the dorsal decubitus position on a warming pad with US transmission gel on the abdomen over the liver. Rats were scanned either with the US probe directly on the abdomen or with the probe above the abdomen using a small water-filled standoff to place the focal zone within the liver. The standoff design used a thin polyethylene window for transmission. When Evans blue dye injection was not used, rats were checked for their heart rate and percentage of oxygen saturation of hemoglobin in peripheral blood, which averaged $325 \pm 18$ beats per minute and $81 \% \pm 3 \%$, respectively.

\section{Diagnostic Ultrasound}

Yang et $\mathrm{al}^{20}$ used a GE Vivid 7 Dimension machine with an S4 transducer placed on the abdomen. The second harmonic B-mode was used with $1.5-\mathrm{MHz}$ transmit and $3.2-\mathrm{MHz}$ receive frequencies. A $3-\mathrm{cm}$ image and focus depth were used with an intermittent exposure scheme. Low-MI images were used to visualize the arrival of contrast agent microbubbles, and then the mode was switched to the flash mode at the maximum MI of 1.0. The high and low modes were switched rapidly to observe alternating phases of microbubble destruction and refill in the imaging plane. The total scan time was 10 minutes.

For this study, an $3 S$ phased array probe (GE Vivid 7 Dimension) was used with two different procedures to exposure the right medial lobe of the liver in a transverse plane. For contact exposure, a $3-\mathrm{cm}$ image and focal depth were used. Our machine had an octave mode with $1.6-\mathrm{MHz}$ transmit and 3.2- $\mathrm{MHz}$ receive frequencies but did not have a flash mode. The machine was therefore switched quickly $(\approx 20$-second cycle time for refill) between a low MI of 0.1 and a maximum MI of 1.0 at 25.2 frames per second, which continued for 10 minutes. This contact method was somewhat inconsistent because of the manual operation. For standoff exposure (SE), the image depth was $8 \mathrm{~cm}$; the focus depth was $5 \mathrm{~cm}$; and the anterior liver lobe was located at the measured optimal focus of $3.8 \mathrm{~cm}$ by adjusting the position of the probe. This arrangement was previously used for studies of contrast-related bioeffects in the rat heart. ${ }^{17}$ The scan pulse repetition frequency was $4.1 \mathrm{kHz}$, and the pulse duration was 1.52 microseconds. A time trigger signal at $1 \mathrm{~Hz}$ was directed into the electrocardiographic input and used to set a 10-second intermittent scan interval. The intermittent triggering allowed refill of the tissue with the contrast agent between triggered dual images. This dual-image method can display contrast microbubble destruction as a loss of contrast enhancement for the second image.

The pulse-pressure waveforms were measured in a water bath using a calibrated hydrophone with a 0.2-mm sensitive spot (HGL0200; Onda, Sunnyvale, $\mathrm{CA})$, and the peak rarefactional pressure amplitude was determined at two depths $0.5 \mathrm{~cm}$ apart, which approximately corresponded to the anterior and posterior surfaces of the scanned liver lobe. The depths were 0.5 and $1.0 \mathrm{~cm}$ for the contact exposure condition and $3.8 \mathrm{~cm}$ and $4.3 \mathrm{~cm}$ for the standoff exposure condition. Values are listed in Table 1 for the pulse parameters and $-6-\mathrm{dB}$ scan widths and thicknesses. For comparison, the dimensions of the active region of the probe were $1.5 \mathrm{~cm}$ (perpendicular to the scan plane) and $2.1 \mathrm{~cm}$ (in the scan plane). Pulse durations were calculated from the time-intensity integral of the pulse waveform. The intermittent scans were performed at the maximum power setting of $0 \mathrm{~dB}$ (MI, 1.3). The standoff method with intermittent imaging was superior to the contact method both in 
Table 1. Results for Measurement of US Pulse Parameters in a Water Bath

\begin{tabular}{|c|c|c|c|c|c|c|}
\hline Method & $\begin{array}{l}\text { Test Depth, } \\
\text { cm }\end{array}$ & $\begin{array}{l}\text { Pulse Duration, } \\
\mu \mathrm{s}\end{array}$ & $\begin{array}{l}\text { PRPA, } \\
\mathrm{MPa}\end{array}$ & $\begin{array}{l}\text { PRPA } / f^{1 / 2}, \\
\text { MPa/MHz }\end{array}$ & $\begin{array}{c}\text { Thickness, } \\
\text { mm }\end{array}$ & $\begin{array}{l}\text { Length, } \\
\text { cm }\end{array}$ \\
\hline Contact & 0.5 & 1.6 & 0.86 & 0.70 & 12.7 & 1.55 \\
\hline Contact & 1.0 & 1.9 & 1.12 & 0.90 & 10.0 & 1.95 \\
\hline Standoff & 3.8 & 1.5 & 2.47 & 2.0 & 5.0 & 6.9 \\
\hline Standoff & 4.3 & 1.5 & 2.40 & 1.9 & 5.0 & 7.7 \\
\hline
\end{tabular}

The contact method used 3-cm focus and depth settings with 25.2 frames per second, whereas the standoff method used a 5 -cm focus and an 8-cm depth with 56.3 frames per second. The scan plane area was determined as -6-dB positions crossing the scan plane (thickness) and along the scan plane (length). PRPA indicates peak rarefactional pressure amplitude.

terms of the consistency of exposure and in terms of the maximum pulse parameters delivered. The contact method was only used for a few tests with Evans blue.

\section{Ultrasound Contrast Agent}

The US contrast agent was a laboratory replacement for Definity, which is no longer sold for research purposes. A replacement contrast agent for Definity was created by using a formula mimicking the Definity formula, as described previously. ${ }^{6}$ The lipid mixture was sterilized and aliquoted into empty sterile Definity vials with the headspace filled with octafluoropropane (HC-218, PurityPlus; Metro Welding Supply, Detroit, MI). The vials were shaken for 45 seconds in a VialMix (DuPont Pharmaceuticals Co, Billerica, MA) before use to produce the suspensions of stabilized microbubbles. The replacement contrast agent microbubbles were assessed with a Coulter counter (Multisizer 4; Beckman Coulter, Inc, Indianapolis, IN) ${ }^{17}$ The mean diameter was slightly larger at $1.8 \pm 0.11 \mu \mathrm{m}$ diameter than actual Definity microbubbles at $1.6 \pm 0.04 \mu \mathrm{m}$, but the concentration of microbubbles in our tests was not substantially different from that of Definity at $3.7 \pm 0.310^{9} \mathrm{~mL}^{-1}$. The replacement contrast agent was prepared each day, diluted with sterile saline, and administered as a bolus dose of $500 \mu \mathrm{L} / \mathrm{kg}$ to reproduce the dosage used by Yang et $\mathrm{al}^{20}$ or as an infusion of $50 \mu \mathrm{L} / \mathrm{kg} / \mathrm{min}$ for the 10-minute scans. Bolus injection was used for contact exposures. For standoff exposures, both bolus dosing and infusion dosing were used in different groups. These dosages were high compared to clinically recommended doses of Definity $(10-\mu \mathrm{L} / \mathrm{kg}$ bolus or $\approx 3.7-\mu \mathrm{L} / \mathrm{kg} / \mathrm{min}$ infusion for a $70-\mathrm{kg}$ human). For sham exposure, the rat was scanned for 5 minutes at the maximum US $(0 \mathrm{~dB})$ setting, and then the contrast agent was infused for 10 minutes with the US off. Examples of the dual images are shown in Figure 1 before and after contrast delivery. The bolus injection produced large contrast enhancement at the surface but shadowing, indicative of increased attenuation, with depth.

\section{Experimental Plan and Measured End Points}

In preliminary testing, the US scanning geometry was established so that the scan plane was located at about the same position for each rat. However, there was no identifiable indication of a contrast-enhanced US-induced effect on the scanned liver samples, in contrast to the clear indications of petechiae, which can be shown in the heart ${ }^{22}$ or kidney. ${ }^{23}$ Groups of rats had blood samples taken before and 30 minutes after scanning for evaluation of liver enzymes, in an effort to detect liver injury, as reported by Yang et al. ${ }^{20}$ Alanine aminotransferase and AST were both measured (Activity assay kits; Sigma-Aldrich, St Louis, MO). The liver was perfused with saline and then with neutral buffered formalin for fixation. Histologic slides were made of tissue slabs cut to crosssection the scan plane to search for injury.

Yang et $\mathrm{al}^{20}$ used Evans blue dye to test for microvascular leakage. In our studies of bioeffects of myocardial contrast echocardiography, Evans blue has been useful for showing microvascular leakage ${ }^{12}$ and also for staining lethally injured cardiomyocytes. ${ }^{23}$ The cardiomyocyte staining can be evaluated by fluorescence microscopy to score numbers of cavitational microlesions, which consist of a petechial hemorrhage and one or more injured cardiomyocytes. ${ }^{17}$ Evans blue dye was prepared at $50 \mathrm{mg} / \mathrm{mL}$ in saline and injected before US scanning at $50 \mathrm{mg} / \mathrm{kg}$. Liver samples were obtained 30 minutes after exposure after perfusion clearance of the circulation with $300 \mathrm{~mL}$ of 
Figure 1. Dual US images during exposure using the standoff method with bolus injection (top row) and infusion (bottom row) of the contrast agent. The left column shows the preinjection images with the anterior liver lobe about $5 \mathrm{~mm}$ thick just below the abdominal wall. Shadows are produced by the spine and by intestinal gas. When the contrast agent is administered (middle column), the image brightness is enhanced at the anterior liver, with attenuation shadowing of deeper tissues, especially for the bolus injection. At the end of the 10-minute exposure (right column), the contrast effect is greatly reduced for the bolus injection but still present for the infusion. The contrast brightness is somewhat reduced for the right image of each pair due to microbubble destruction.

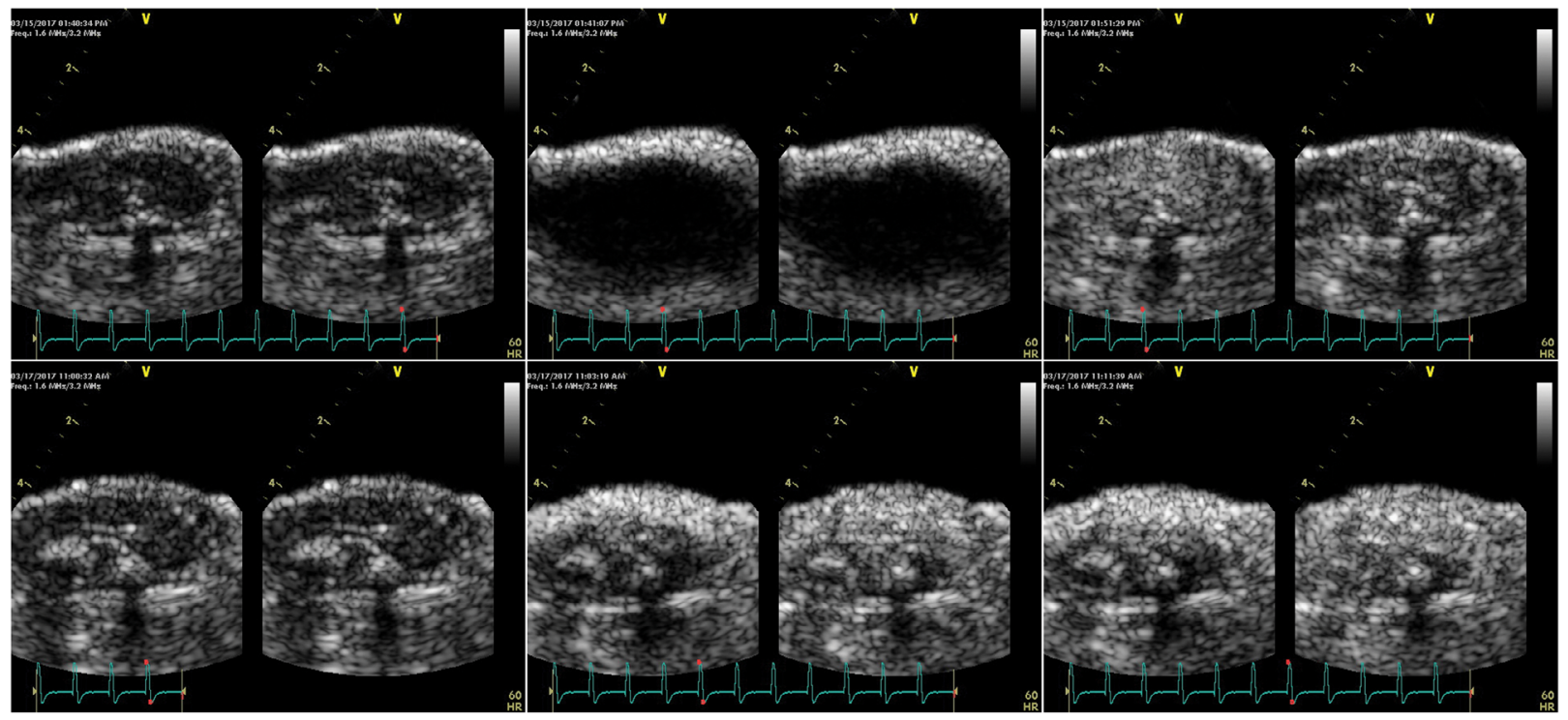

heparin saline. For evaluation of microvascular leakage, liver samples were weighed, minced, and extracted in formamide. After centrifugation, the supernatant was measured by spectrophotometry at $620 \mathrm{~nm}$ to determine the Evans blue content. In addition, blue-stained cells were evident on the liver surface within the scan plane. This effect was scored in frozen sections by fluorescence microscopy. Three sections cut to cross-section the scan plane (spaced $1.5 \mathrm{~mm}$ apart) from each rat liver were scored by counting fluorescent cells in 5 adjacent $\times 10$ fields of view from the anterior surface inward to the posterior surface of the sample. The scoring was performed on photomicrographic images (Spot Flex; Diagnostic Instruments, Inc, Sterling Heights, MI) for fixed exposure times set to maximize the conspicuity of the stained cells (relative to the background).

The Evans blue appeared to stain the cytoplasm of hepatocytes, which was not clearly indicative of lethal injury. A second method of vital staining was tried using a trypan blue vital stain, based on staining methods for liver injury. ${ }^{24}$ After perfusion with heparin saline, perfusion was continued with $120 \mathrm{~mL}$ of trypan blue solution (10:1 dilution by heparin saline of $0.4 \%$ trypan blue stain; Gibco, Fisher Scientific, Hampton, NH) followed by $120 \mathrm{~mL}$ of heparin saline. The liver samples showed stained cells in the scan plane after this procedure. Histologic observation was also tried using perfusion and immersion fixation with neutral buffered formalin and preparation of histologic paraffin sections without only the eosin stain, as reported previously. ${ }^{24}$ However, the histologic slides did not retain the trypan blue staining sufficiently for confident scoring, and only a qualitative observation of fresh samples was performed.

The numbers of rats used for the different exposures and tests are listed in Table 2. Results were evaluated by comparisons of two means of measurements in different groups of rats. Statistical analysis was performed with SigmaPlot version 11.0 software for Windows (Systat Software, Inc, San Jose, CA). The Mann-Whitney rank sum test and the Student $t$ test were used for the comparisons of unrelated samples, whereas pared tests were used for comparing beforeand-after tests. Statistical significance was assumed at $P<.05$. 
Table 2. Numbers of Rats Used for the Various Exposures and Evaluation Tests

\begin{tabular}{|c|c|c|c|c|c|c|}
\hline \multirow[b]{2}{*}{ Test } & \multicolumn{2}{|c|}{ Bolus } & \multicolumn{2}{|c|}{ Bolus } & \multicolumn{2}{|c|}{ Infusion } \\
\hline & CE & CE Sham & SE & SE Sham & SE & SE Sham \\
\hline Enzymes & NP & NP & 7 & 5 & 6 & 5 \\
\hline Evans blue extraction & 6 & 6 & 8 & NP & 8 & 6 \\
\hline Evans blue count & 4 & 4 & 6 & NP & 11 & 10 \\
\hline Trypan blue & NP & NP & 6 & NP & 6 & NP \\
\hline
\end{tabular}

CE indicates contact exposure; NP, not performed; and SE, standoff exposure.

\section{Results}

Enzyme assays were performed for the standoff method using bolus and infusion contrast delivery. Results for ALT are shown in Figure 2 as before-andafter comparisons measured in sham and exposed samples. The ALT for exposure with contrast infusion was significantly elevated relative to shams $(P<.05)$. For AST, the enzyme activity was significantly increased for both contrast infusion and bolus, and the after-exposure results was significantly elevated above the before-exposure results for the contrast bolus (Figure 3). The enzyme results showed enzyme release indicative of liver cell injury.

The extraction of Evans blue dye from exposed liver samples was performed for all of the methods. Results are shown in Figure 4. Only the exposure with contrast infusion was significantly increased $(P<.05)$ above both its respective control samples (out of the beam path) and sham-exposed samples.

Figure 2. Results for measurements of plasma ALT in blood samples taken before and after sham or exposure. The ALT was significantly increased $(P<.05)$ for the after-exposure sample with infusion (\#) relative to the sham.

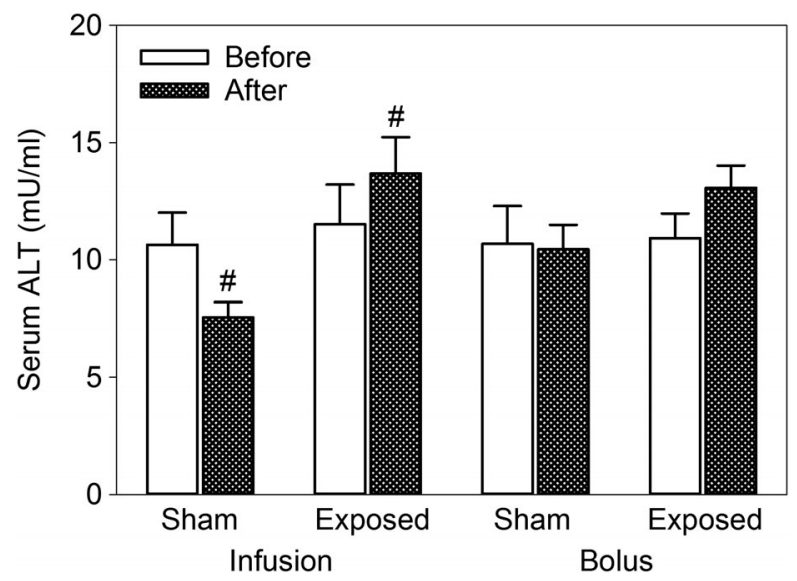

These results indicated that the contact exposure method was relatively ineffective. In addition, the standoff exposure with contrast infusion appeared to be more effective than exposure with the bolus injection of the contrast agent.

In preliminary tests, freshly excised livers were examined and fixed for histologic analysis with hematoxylin-eosin staining to find any clear indications of injury, such as petechiae, which are seen for CEDUS of other organs. No bioeffects could be clearly identified, even after perfusion clearance of the blood in the circulation. However, both the Evans blue and the trypan blue methods revealed a band of scattered stained cells corresponding to the scan plane in fresh samples. Outside this region, stained cells were essentially absent, confirming the effect of US exposure in the scan plane. Examples of the appearance of the freshly excised livers are shown in

Figure 3. Results for measurements of plasma AST in blood samples taken before and after sham or exposure. The AST was significantly elevated $(P<.05)$ for the after-exposure sample with infusion relative to sham $(\#)$ and for the bolus injection for the exposed sample relative to both the sham (\#) and the beforeexposure sample (*).

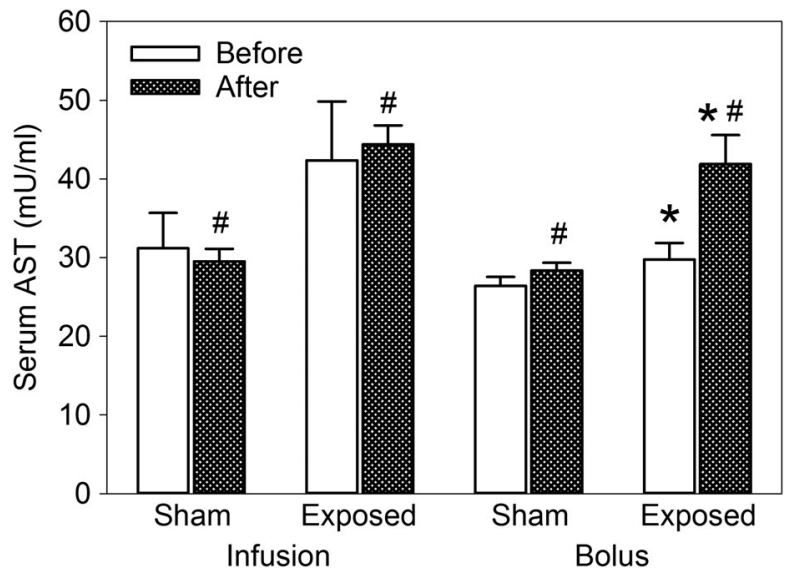


Figure 4. Results for the extraction of Evans blue from liver samples. For the standoff exposure with infusion of the contrast agent $(*)$, the extracted Evans blue was significantly elevated $(P<.05)$ relative to paired controls and to shams.

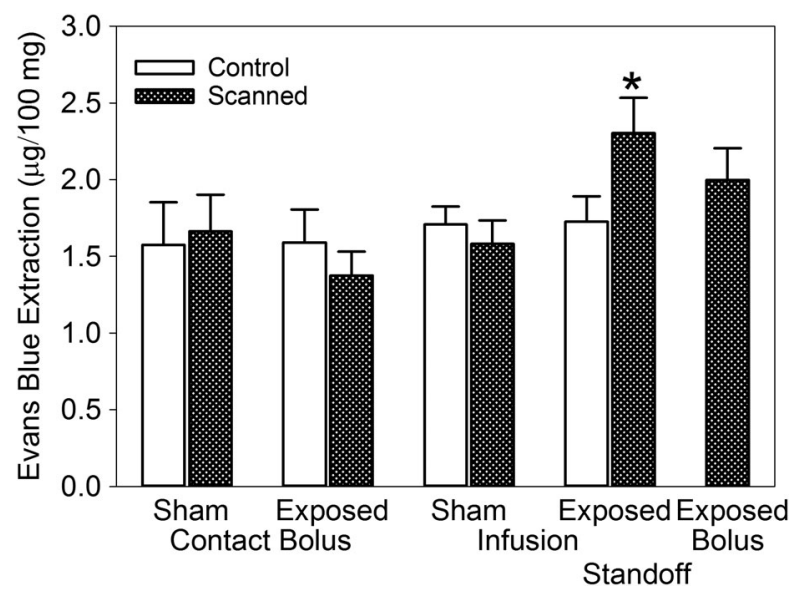

Figure 5. The Evans blue-stained cells showed low contrast in the bright field, but the trypan blue gave a readily discernable bluish band, which resulted from numerous blue-stained cells, in the scan plane.

In frozen sections, the Evans blue-stained cells could be readily identified by the red fluorescence of the Evans blue, as shown in Figure 6. There was a substantial background in some samples, in which cells were outlined in red fluorescence, possibly due to incomplete perfusion removal of the dye. However, the positively stained cells were distinct with bright fluorescence, which filled in the entire cell. In fresh tissue slices, the individual trypan blue-stained cells were readily identified by strong blue staining of the cell nuclei, as shown in Figure 6 at the same scale as the Evans blue frozen-section image. Qualitatively, the scattered distribution and number of affected cells appeared to be about the same for both staining procedures. Unfortunately, the fresh hand-cut slices were thick and uneven so that the staining methods could not be quantitatively compared. As noted in "Materials and Methods," the histologic processing of the trypan blue-stained samples and staining with eosin only gave greatly diminished conspicuity of the stained cells (compared to fresh samples), and these could not be scored with confidence. The Evans blue-stained cells could be quantitatively counted, and the fluorescent cell counts are presented in Figure 7. The contact exposure method gave relatively low results compared to the standoff exposure bolus method $(P<.01$ for the anterior position). In addition, the standoff exposure method with contrast infusion appeared to be more efficacious relative to the contrast bolus, particularly for deeper portions of the liver sample $(P<.05)$.

\section{Discussion}

Contrast-enhanced diagnostic US has a potential for induction of localized biological effects, which have been reported, for example, in muscles, the heart, and kidneys. The effects include microvascular leakage, petechial hemorrhage, and lethal injury of parenchymal cells at the highest pulse pressure amplitudes. The liver is a good candidate for CEDUS examination, but little research has been reported for

Figure 5. Stereo microscopic images of freshly excised liver lobes after perfusion clearance of blood and the dye solution: Evans blue (top) and trypan blue (bottom). Arrows indicate the position of the blue band of stained cells within the scan plane; and scale bars, $1 \mathrm{~cm}$.

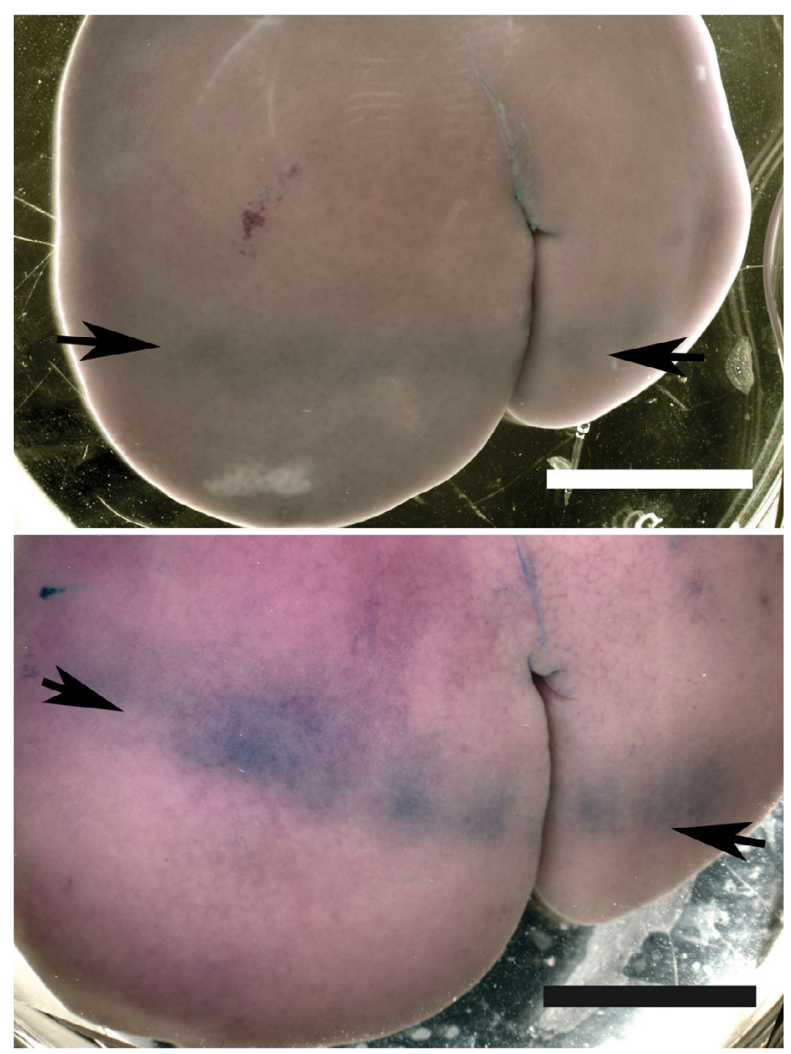


Figure 6. Images of the Evans blue-stained cells for fluorescence microscopy of a frozen section (left) and trypan blue-stained cells for stereomicroscopy of a freshly cut sample (right). Both samples had standoff exposure with contrast infusion. Scale bars indicate $0.5 \mathrm{~mm}$.

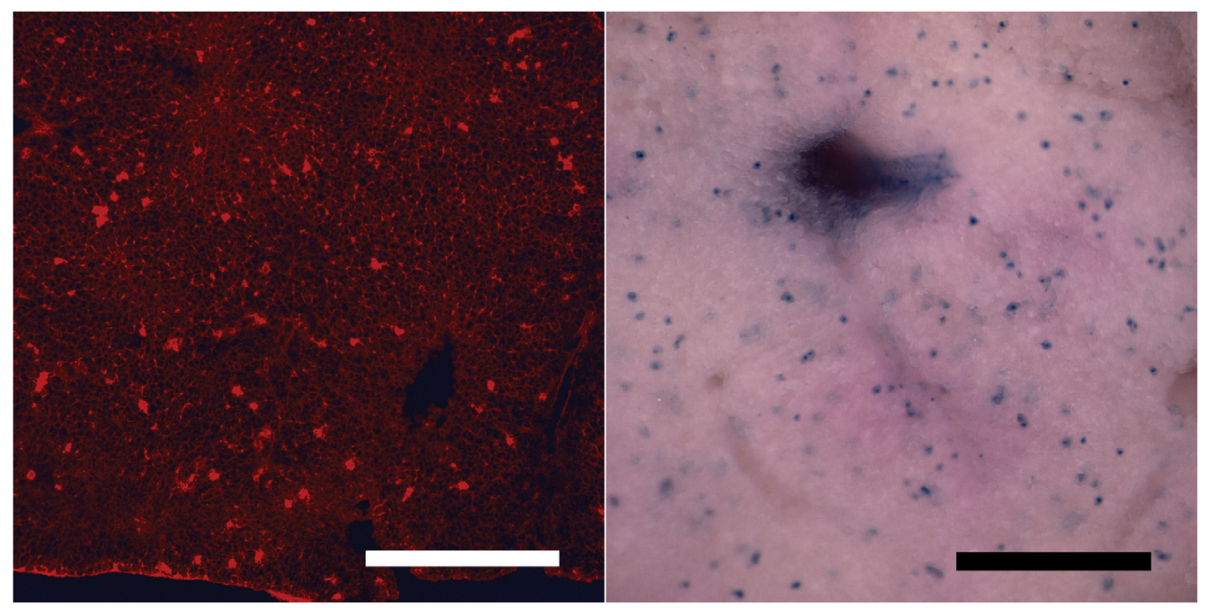

potential bioeffects in the liver. In this study, the potential for bioeffects was researched in rat livers, following a report by Yang et $\mathrm{al}^{20}$ of CEDUS-induced liver enzyme release, and vascular permeabilization.

The methods of Yang et $\mathrm{al}^{20}$ were used as much as possible, although our US machine was not capable of duplicating their exposures. The contact exposure method placed the liver lobe in the near field of the probe and delivered reduced exposure levels

Figure 7. Results for Evans blue-stained cells counted in fluorescent microscopy of frozen sections. Five fields of view were scored for each sample lobe, from the anterior surface $(0 \mathrm{~mm})$ inward toward the posterior side. The strong decrease of the score with depth for the bolus injection and standoff exposure probably reflects the attenuation produced by the high contrast agent dose (see Figure 1).

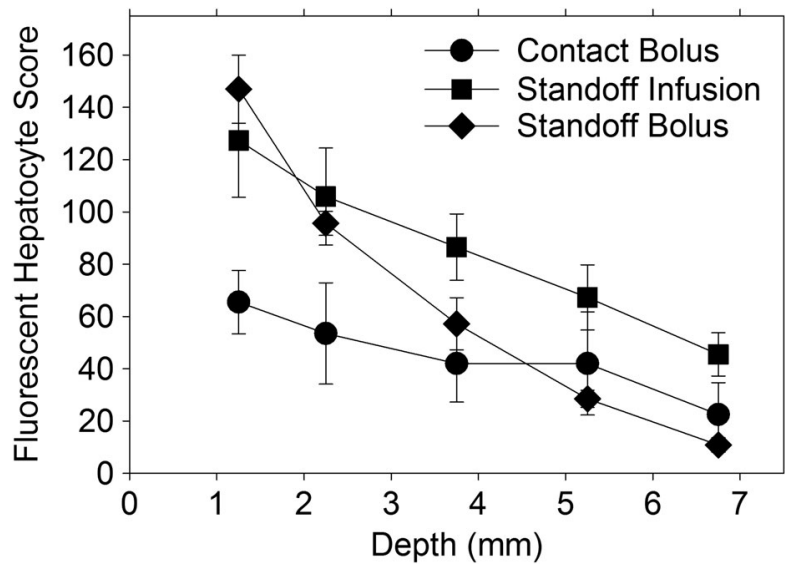

compared to the focal zone (Table 1). Our study included mostly focal zone exposure using a standoff, which provided higher exposure levels in the anterior liver lobe and was similar to previous research in other organs. The large bolus dose produced high attenuation, evident in the US images (Figure 1). In our study, we added exposure with 10-second intermittent scans and infusion of the contrast agent, which reduced attenuation and shadowing.

The results showed enzyme release for 30-minute samples (Figures 2 and 3). In addition, extraction of Evans blue dye from the exposed tissue showed a significant increase for the standoff exposure method within contrast infusion (Figure 4). Therefore, the findings of Yang et $\mathrm{al}^{20}$ were confirmed qualitatively, even though the methods could not be quantitatively compared.

In addition, we found that Evans blue and trypan blue vital-staining methods revealed a band of scattered stained cells corresponding to the US scan plane (Figure 5). Both methods appeared to detect about the same distribution and number of stained cells (Figure 6), which indicates lethal injury of some hepatocytes. The level of injury was small, less than $1 \%$ of cells, but nevertheless quite clearly defined. For the Evans blue method, a key step was perfusion clearance of the blood. Even lengthy perfusion clearance left a substantial background in the form of a fluorescent border around many hepatocytes. This was not a bioeffect of the CEDUS and may simply 
have shown remaining Evans blue, possibly within the space of Disse, which was not cleared by the perfusion blood clearance step. Although the trypan blue method was not amenable to quantitative histologic characterization, the Evans blue method gave quantitative fluorescent cell counts as a function of depth into the liver samples (Figure 7). These results showed a strong decrease with depth for bolus injection, probably reflective of the high attenuation noted for this agent delivery method.

Given the high contrast agent doses, the effects in this study seemed relatively small, for example, compared to the heart. ${ }^{17}$ However, the dose was sufficient to produce substantial attenuation, possibly reducing the exposure impact. In addition, the low microvascular impact may be due to the nature of the sinusoidal capillaries present in the liver, which are large $(7-15 \mu \mathrm{m}$ in diameter) and lined by discontinuous endothelium. ${ }^{25}$ Recent research indicates that the capillaries, for example, in the kidney are injured by the action of the largest microbubbles in contrast agents, and microbubble expansion sufficient to stress capillary walls is required for capillary rupture. ${ }^{26,27}$ The minimal impact on liver sinusoids may be related to the limited number of large microbubble sizes available after passage through the lungs. Further work has been pursued to better define the bioeffect response in terms of both the contrast agent dosage and microbubble sizes and the US pulse amplitude threshold for the hepatocyte injury, and results have been reported in a separate article. ${ }^{28}$

\section{References}

1. Szabo TL. Diagnostic Ultrasound Imaging: Inside Out. 2nd ed. New York, NY: Elsevier; 2014.

2. Claudon M, Dietrich CF, Choi BI, et al; World Federation for Ultrasound in Medicine; European Federation of Societies for Ultrasound. Guidelines and good clinical practice recommendations for contrast enhanced ultrasound (CEUS) in the liverupdate 2012: a WFUMB-EFSUMB initiative in cooperation with representatives of AFSUMB, AIUM, ASUM, FLAUS and ICUS. Ultrasound Med Biol 2013; 39:187-210.

3. D'Onofrio M, Crosara S, De Robertis R, Canestrini S, Mucelli RP. Contrast-enhanced ultrasound of focal liver lesions. AJR Am J Roentgenol 2015; 205:W56-W66.
4. Jang HJ, Kim TK, Burns PN, Wilson SR. CEUS: an essential component in a multimodality approach to small nodules in patients at high-risk for hepatocellular carcinoma. Eur J Radiol 2015; 84: $1623-1635$.

5. Chiorean L, Cantisani V, Jenssen C, Sidhu PS, Baum U, Dietrich CF. Focal masses in a non-cirrhotic liver: the additional benefit of CEUS over baseline imaging. Eur J Radiol 2015; 84: 1636-1643.

6. Miller DL, Dou C, Lu X, et al. The use of theranostic strategies in myocardial cavitation-enabled therapy. Ultrasound Med Biol 2015; 41:1865-1875.

7. Miller DL, Averkiou MA, Brayman AA, et al. Bioeffects considerations for diagnostic ultrasound contrast agents. J Ultrasound Med 2008; 27:611-632.

8. Miller DL, Gies RA. Gas-body-based contrast agent enhances vascular bioeffects of $1.09 \mathrm{MHz}$ ultrasound on mouse intestine. Ultrasound Med Biol 1998; 24:1201-1208.

9. Skyba DM, Price RJ, Linka AZ, Skalak TC, Kaul S. Direct in vivo visualization of intravascular destruction of microbubbles by ultrasound and its local effects on tissue. Circulation 1998; 98:290-293.

10. Miller DL, Quddus J. Diagnostic ultrasound activation of contrast agent gas bodies induces capillary rupture in mice. Proc Natl Acad Sci USA 2000; 97:10179-10184.

11. Chen S, Kroll MH, Shohet RV, Frenkel P, Mayer SA, Grayburn PA. Bioeffects of myocardial contrast microbubble destruction by echocardiography. Echocardiography 2002; 19: 495-500.

12. Li P, Cao LQ, Dou CY, Armstrong WR, Miller DL. Impact of myocardial contrast echocardiography on vascular permeability: an in vivo dose response study of delivery mode, ultrasound power and contrast dose. Ultrasound Med Biol 2003; 29:1341-1349.

13. Wible JH Jr, Galen KP, Wojdyla JK, Hughes MS, Klibanov AL, Brandenburger GH. Microbubbles induce renal hemorrhage when exposed to diagnostic ultrasound in anesthetized rats. Ultrasound Med Biol 2002; 28:1535-1546.

14. Miller DL, Dou C, Wiggins RC, Wharram BL, Goyal M, Williams AR. An in vivo rat model simulating imaging of human kidney by diagnostic ultrasound with gas-body contrast agent. Ultrasound Med Biol 2007; 33:129-135.

15. Miller DL, Dou C, Sorenson D, Liu M. Histological observation of islet hemorrhage induced by diagnostic ultrasound with contrast agent in rat pancreas. PLoS One. 2011; 6:e21617.

16. Miller DL, Dou C, Wiggins RC. Frequency dependence of kidney injury induced by contrast-aided diagnostic ultrasound in rats. Ultrasound Med Biol 2008; 34:1678-1687.

17. Miller DL, Lu X, Fabiilli M, Fields K, Dou C. Frequency dependence of petechial hemorrhage and cardiomyocyte injury induced during myocardial contrast echocardiography. Ultrasound Med Biol 2016; 42:1929-1941. 
18. Shigeta K, Itoh K, Ookawara S, Taniguchi N, Omoto K. Endothelial cell injury and platelet aggregation induced by contrast ultrasonography in the rat hepatic sinusoid. J Ultrasound Med 2004; 23: 29-36.

19. Shigeta $\mathrm{K}$, Itoh $\mathrm{K}$, Ookawara $\mathrm{S}$, Taniguchi $\mathrm{N}$, Omoto $\mathrm{K}$. The effects of Levovist and DD-723 in activating platelets and damaging hepatic cells of rats. J Ultrasound Med 2005; 24: 967-974.

20. Yang D, Tan KB, Gao YH, Liu H, Yang WX. Effects of diagnostic ultrasound-targeted microbubble destruction on permeability of normal liver in rats. Ultrasonics 2012; 52:1065-1071.

21. Liu P, Wang X, Zhou S, Hua X, Liu Z, Gao Y. Effects of a novel ultrasound contrast agent with long persistence on right ventricular pressure: comparison with SonoVue. Ultrasonics 2011; 51: 210-214.

22. Miller DL, Li P, Gordon, D, Armstrong WF. Histological characterization of microlesions induced by myocardial contrast echocardiography. Echocardiography 2005; 22:25-34.

23. Miller DL, Li P, Dou C, Armstrong WF, Gordon D. Evans blue staining of cardiomyocytes induced by myocardial contrast echocardiography in rats: evidence for necrosis instead of apoptosis. Ultrasound Med Biol 2007; 33:1988-1996.

24. Belinsky SA, Popp JA, Kauffman FC, Thurman RG. Trypan blue uptake as a new method to investigate hepatotoxicity in periportal and pericentral regions of the liver lobule: studies with allyl alcohol in the perfused liver. J Pharmacol Exp Ther 1984; 230:755-760.

25. Vollmar B, Menger MD. The hepatic microcirculation: mechanistic contributions and therapeutic targets in liver injury and repair. Physiol Rev 2009; 89:1269-1339.

26. Church CC, Miller DL. A two-criterion model for microvascular bioeffects induced in vivo by contrast microbubbles exposed to medical ultrasound. Ultrasound Med Biol 2016; 42:1385-2398.

27. Miller DL, Lu X, Dou C, Fabiilli ML, Church CC. The dependence of glomerular capillary hemorrhage induced by contrast enhanced diagnostic ultrasound on microbubble diameter. Ultrasound Med Biol 2018; 44:613-621.

28. Miller DL, Lu X, Fabiilli M, Dou C. Influence of microbubble size and pulse amplitude on hepatocyte injury induced by contrast-enhanced diagnostic ultrasound. Ultrasound Med Biol 2018; Oct 23. pii: S03015629(18)30409-5. doi: 10.1016/j.ultrasmedbio.2018.09.011. 